\title{
Continuous Solitons in a Lattice Nonlinearity
}

\author{
D. Pierangeli, ${ }^{1,{ }^{*}}$ M. Flammini, ${ }^{1}$ F. Di Mei, ${ }^{1,2}$ J. Parravicini, ${ }^{1,3}$ C. E. M. de Oliveira, ${ }^{4}$ A. J. Agranat, ${ }^{4}$ and E. DelRe ${ }^{1,3}$ \\ ${ }^{1}$ Dipartimento di Fisica, Università di Roma "La Sapienza", 00185 Rome, Italy \\ ${ }^{2}$ Center for Life Nano Science@Sapienza, Istituto Italiano di Tecnologia, 00161 Rome, Italy \\ ${ }^{3} I P C F-C N R$, Università di Roma "La Sapienza", 00185 Rome, Italy \\ ${ }^{4}$ The Brojde Center for Innovative Engineering and Computer Science, The Hebrew University, Jeruslaem 91904, Israel
}

(Received 17 December 2014; published 20 May 2015)

\begin{abstract}
We study theoretically and experimentally the propagation of optical solitons in a lattice nonlinearity, a periodic pattern that both affects and is strongly affected by the wave. Observations are carried out using spatial photorefractive solitons in a volume microstructured crystal with a built-in oscillating low-frequency dielectric constant. The pattern causes an oscillating electro-optic response that induces a periodic optical nonlinearity. On-axis results in potassium-lithium-tantalate-niobate indicate the appearance of effective continuous saturated-Kerr solitons, where all spatial traces of the lattice vanish, independently of the ratio between beam width and lattice constant. Decoupling the lattice nonlinearity allows the detection of discrete delocalized and localized light distributions, demonstrating that the continuous solitons form out of the combined compensation of diffraction and of the underlying periodic volume pattern.
\end{abstract}

DOI: 10.1103/PhysRevLett.114.203901

PACS numbers: 42.65.Tg, 05.45.Yv, 42.70.Nq, 42.70.Qs

The coupling between different and matched spectral components of the optical field is one of the fundamental effects governing propagation through periodic systems [1]. The interplay between this coherent effect and nonlinearity has been extensively investigated allowing diffraction control $[2,3]$ and giving rise to self-localized states, such as discrete and gap solitons [4-9]. Experiments on discrete trapping are generally based on photonic lattices made from etched waveguide arrays [5,8] or created through optical induction in photorefractive media $[7,10]$. Studies have spanned a wide variety of physical mechanisms affecting solitons, such as unconventionally biasing [11,12], PT symmetry [13], and disorder [14]. At present, however, the soliton has always evolved in a fixed linear or nonlinear pattern, i.e., in conditions in which the lattice is not appreciably affected by the wave $[3,15]$.

Here, we study an entirely opposite condition: spatial solitons that form in a lattice nonlinearity. A lattice nonlinearity is a periodic variation in the nonlinear response that is, in turn, negligible in the linear response. This means the lattice itself depends on the soliton, and both lattice and soliton are strongly interacting during propagation. This fundamental difference with respect to previous experiments is schematically illustrated in Fig. 1, where the optical propagation in a photonic lattice is compared with that in a lattice nonlinearity. In general, the standard physical condition [Fig. 1(a)] consists of a medium with a periodic index of refraction variation $\delta n_{\text {latt }}$, affecting, parametrically, the superimposed soliton nonlinearity $\delta n_{\text {sol }}$. So, while the nonlinear waves evolve into a latticedependent trapped state, $\delta n_{\text {latt }}$ remains almost completely unaffected by the waves' dynamics. On the contrary, if the beam and lattice are mutually nonlinear, $\delta n=$ $\delta n\left(\delta n_{\text {sol }}, \delta n_{\text {latt }}\right)$, the nonlinear propagation spatially modifies the underlying periodic pattern itself [Fig. 1(b)].

In our present experiment, we report the first observation of continuous solitons in a lattice nonlinearity. The effect is achieved in a microstructured photorefractive ferroelectric crystal. The lattice nonlinearity emerges through the strong interaction of the localized nonlinearity $\delta n_{\text {sol }}$ and the delocalized periodic nonlinearity $\delta n_{\text {latt }}$ driven by the photoinduced space-charge field. The resulting on-axis propagation dynamically shows a transition from a discrete pattern to a soliton with the peculiar property of being continuous in the transverse dimension, irrespective of the
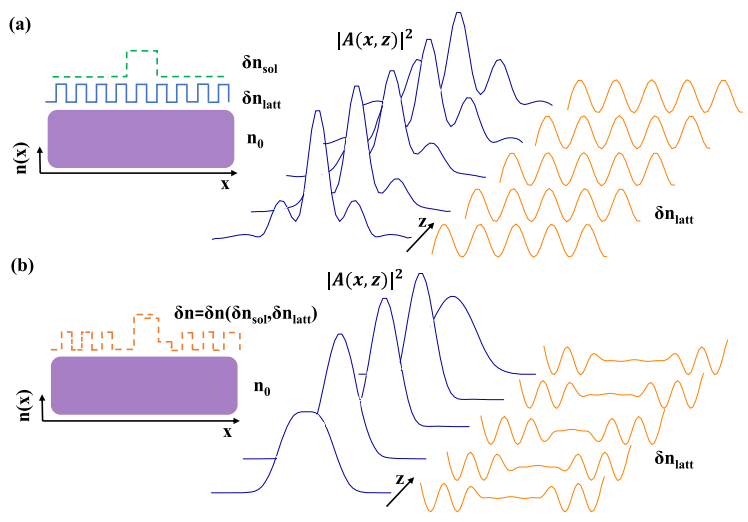

FIG. 1 (color online). Nonlinear propagation in periodic systems. (a) Trapping in photonic lattices: the periodic pattern $\delta n_{\text {latt }}$ affects the spatial propagation but is not affected by the wave. (b) Trapping in lattice nonlinearity: optical field and lattice are mutually coupled and $\delta n_{\text {latt }}$ depends on the waveform. 
beam size compared to the grating period. Discrete properties also emerge dynamically in the zero field after the steady-state soliton formation, when the electro-optic and photorefractive responses are decoupled.

We consider a compositionally disordered photorefractive potassium-lithium-tantalate-niobate (KLTN) crystal, $\mathrm{K}_{1-\alpha} \mathrm{Li}_{\alpha} \mathrm{Ta}_{1-\beta} \mathrm{Nb}_{\beta} \mathrm{O}_{3}$ with $\alpha=0.04$ and $\beta=0.38$, grown through the top-seeded solution method by extracting a zero-cut $2.4^{(x)} \times 2.0^{(y)} \times 1.7^{(z)} \mathrm{mm}$ optical quality specimen. The Curie point at the temperature $T_{C}=294 \mathrm{~K}$ is measured and characterized through low-frequency dielectric spectroscopy that also signals the absence of large deviations from the mean-field behavior, typical of other near-transition disordered ferroelectric samples [16,17]. The sample is grown so as to manifest a sinusoidal variation in the low-frequency dielectric constant [18-20]. An electric field can turn this volume microstructure into a periodic index of refraction modulation $\Delta n(x)$ through the quadratic electro-optic effect [21]. The lattice nonlinearity arises when this electric field is optically induced, as occurs for the photorefractive screening nonlinearity $[22,23]$. The leading terms are

$$
\begin{aligned}
\Delta n(x)= & \delta n_{0} \cos (K x) \\
& -\frac{1}{2} n_{0}^{3} g_{\mathrm{eff}} \varepsilon_{0}^{2} \varepsilon_{r}^{2}\left[1+\frac{2 \delta T_{c}}{T-T_{C}} \cos (K x)\right]\left(\frac{E_{0}}{1+u(x)^{2}}\right)^{2},
\end{aligned}
$$

where $\delta n_{0}$ contains the contribution of the Sellmeier's refractive index grating, $n_{0}$ is the unperturbed average index of refraction, $g_{\text {eff }}$ the appropriate electro-optic coefficient, $\delta T_{c}$ the amplitude of the nonlinear grating,
$K=2 \pi / \Lambda$ the grating number, $\Lambda$ being the grating period, $E_{0}=V / l_{x}\left(l_{x}=2.4 \mathrm{~mm}\right)$ the bias field amplitude, and $u(x)=\sqrt{I(x) / I_{B}}$ the beam intensity normalized to that of the background. It follows that for a $(1+1 \mathrm{D})$ paraxial monochromatic beam, propagating on axis in the presence of an electro-optic lattice nonlinearity, the envelope $A(x, z)$ of the optical field satisfies the nonlinear parabolic equation

$$
i \frac{\partial A(x, z)}{\partial z}+\frac{1}{2 k} \frac{\partial^{2} A(x, z)}{\partial x^{2}}=-\frac{k}{n_{0}} \Delta n(x) A(x, z),
$$

with $\Delta n(x)$ expressed according to Eq. (1) and $k=2 \pi n_{0} / \lambda$. Numerical results are obtained using the beam propagation method to resolve this equation with parameters that match those from our experiment. Referring to Eq. (1), it should be noted that the first linear term only weakly affects propagation. It is central to our present study that, in turn, the field-dependent lattice term is not at all a perturbation to the screening nonlinearity. Indeed, in our sample, we obtain from the measured Bragg diffraction efficiency [19] $\delta n_{0} \approx 5 \times 10^{-5}$ and $\delta T_{C} \approx 1 \mathrm{~K}$, working at $T=T_{C}+4 \mathrm{~K}$ as in Fig. 3. Other parameters are $n_{0}=2.4$, $\varepsilon_{r}=0.91 \times 10^{4}$, and $g_{\text {eff }}=0.16 \mathrm{~m}^{2} / \mathrm{C}^{4}$.

The experimental geometry in which focused beams are launched into the microstructured KLTN is shown in Fig. 2(a); $\mu \mathrm{W}$ cylindrical waves at $\lambda=532 \mathrm{~nm}$ propagate in a transmission configuration with respect to the grating, with the main wave vector $\mathbf{k}=\mathbf{k}_{z}$ orthogonal to the grating vector $\mathbf{K}$ and to the bias field (on axis, $\vartheta=0$ ). Light beam polarization is chosen to maximize the external field effect. The grating is optically detected with a plane wave transmitted at the resonant Bragg condition $\vartheta=\vartheta_{B}$
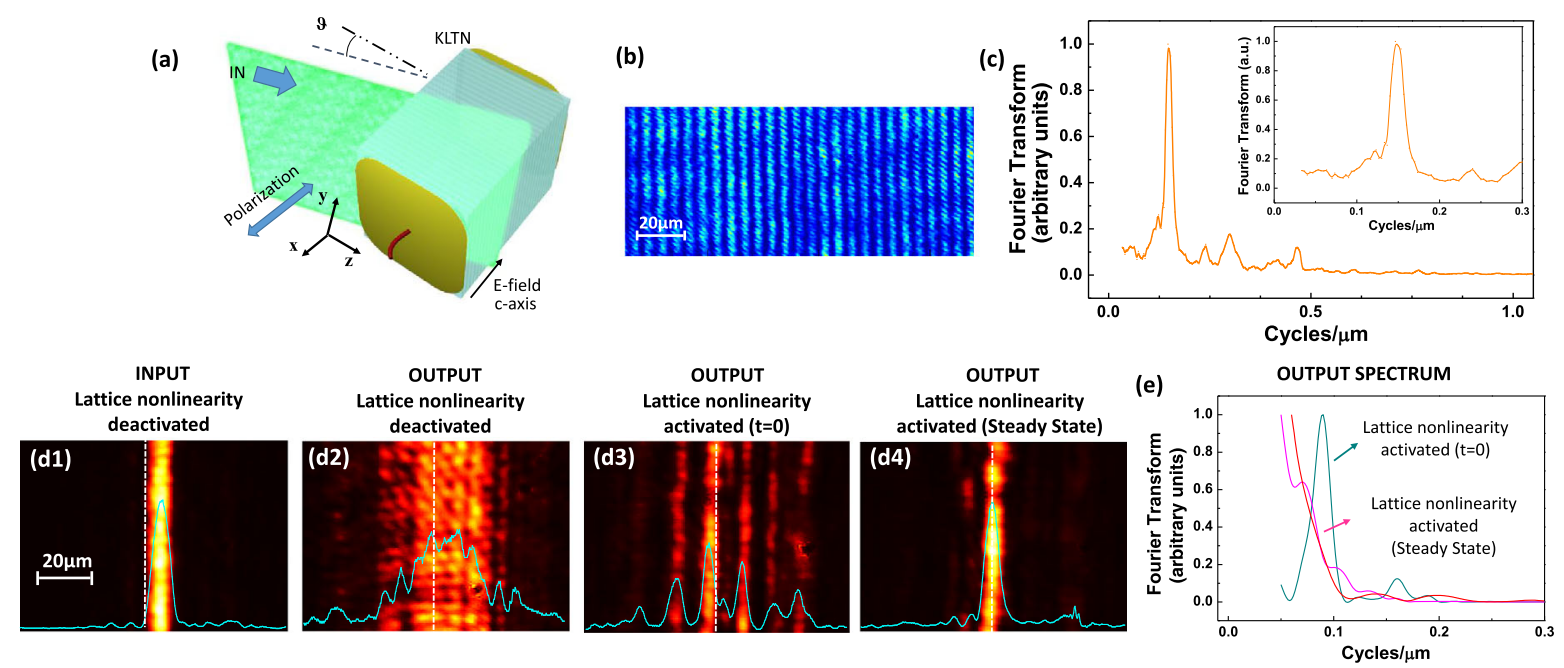

FIG. 2 (color online). Light propagation in the lattice nonlinearity embedded in a microstructured KLTN. (a) Sketch of the physical geometry and (b)-(c) optical lattice detection: (b) transmission microscopy image at $\vartheta=\vartheta_{B}$ and (c) its intensity Fourier transform revealing the grating period $\Lambda=5.5 \pm 0.3 \mu \mathrm{m}$ (expanded view in the inset). (d) Observed beam dynamics from the starting delocalized discrete pattern to the continuous soliton. (d1) Input and (d2) output beam when the lattice nonlinearity is deactivated. (d3) Output discrete spatial distribution as soon as the lattice nonlinearity is enabled at $V=400 \mathrm{~V}$ and (d4) continuous soliton at the steady state. (e) Intensity Fourier transform of (d2) (red line), (d3) (cyan line), and (d4) (magenta line). 
[Figs. 2(b)-2(c)]; the Fourier transform reveals a grating period $\Lambda=5.5 \pm 0.3 \mu \mathrm{m}$.

The observed beam propagation dynamics inside the microstructured nonlinear lattice is shown in Fig. 2(d). When the electro-optical response is not activated through the bias field, the input Gaussian beam with full width at half maximum $\mathrm{FWHM}=7 \mu \mathrm{m}$ experiences quasilinear diffraction, resulting in a FWHM $=26 \mu \mathrm{m}$ output distribution [Figs. 2(d1)-2(d2)]. In these conditions, only the weak linear part of the lattice is involved in the beam propagation and, analyzing the spectrum [Fig. 2(e)], its affect on the beam is negligible. However, when the beam is exposed to the lattice nonlinearity, that is, the sample is biased, it instantaneously (at fast electro-optic response times) rearranges itself over the periodic index of refraction pattern. Considering the $\mu \mathrm{W}$ power used in the experiments, the photorefractive response begins changing the pattern only approximately 10-20 seconds after this initial stage. The discrete light distribution emerging in the first instants, before the light is able to produce the space-charge field, is shown in Fig. 2(d3), for $u_{0}=\sqrt{I_{\text {peak }} / I_{B}} \simeq 8$ and $V=400 \mathrm{~V}$, and appears delocalized compared to the input beam distribution. The operational temperature in this case is $T=T_{C}+2 \mathrm{~K}$, so that, from Eq. (1), we expect a nonlinear lattice with the same amplitude of the "homogeneous" photorefractive nonlinearity ( $\Delta n$ of the order of $10^{-3}$ ). The buildup of the space-charge field causes the progressive local screening of the periodic lattice until the continuous steady-state soliton forms after a few minutes
[Fig. 2(d4)]. The soliton transverse profiles have no trace of a periodic feature, so the lattice nonlinearity allows the transition from a discrete delocalized pattern to a continuous soliton. In Fourier space (transverse spatial spectrum), the spectrum of the output intensity distribution passes from having a dominant peak compatible with the lattice spatial frequency to a monotonic decaying behavior without dominant resonances [Fig. 2(e)].

The continuous soliton behavior in the lattice nonlinearity is demonstrated in Fig. 3. When no bias field is applied, the input beam with FWHM $=7 \mu$ m experiences homogeneous diffraction resulting in an FWHM $=24 \mu \mathrm{m}$ output distribution [Figs. 3(a1)-3(b1)]. Applying a $V=580 \mathrm{~V}$ static potential, a steady-state soliton propagation is obtained for an intensity ratio $u_{0} \simeq 5$ [Fig. 3(c1)]. This absence of discrete features is confirmed by numerical simulation revealing a soliton shape typical of continuous solitons, as reported in Fig. 3(d1). Interestingly, this finding does not match what is expected for photorefractive solitons in a fixed bulk grating, where waveforms satisfying a two-parameter existence condition are characterized by modulated components [15]. This underlines the role of the lattice nonlinearity. The continuous behavior has roots in the coupling between periodic and nonperiodic terms in the soliton supporting nonlinearity. Indeed, simulations demonstrate an index of refraction variation losing the sinusoidal behavior in the soliton region [Fig. 3(f1)]; the screening field locally leads the underlying lattice into a latent state. This effect is independent both of the grating
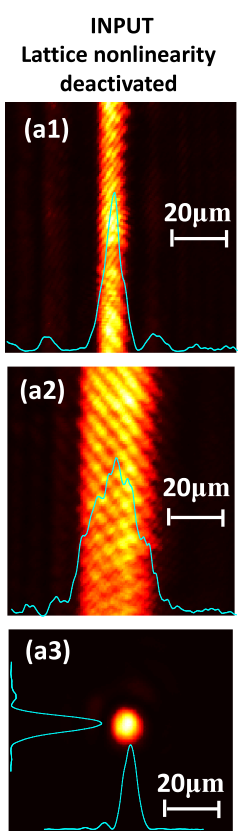
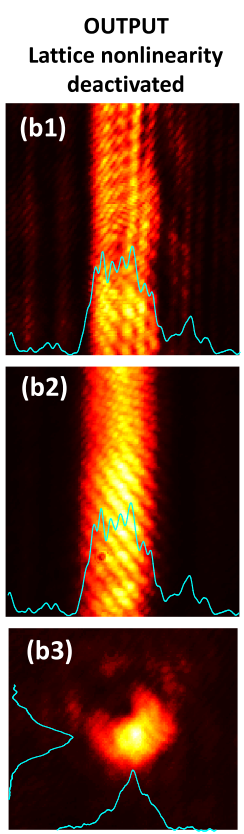
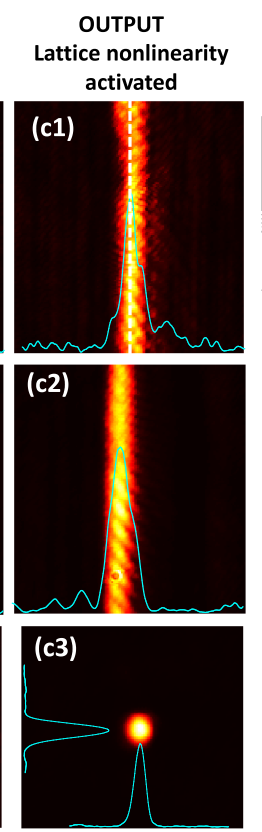
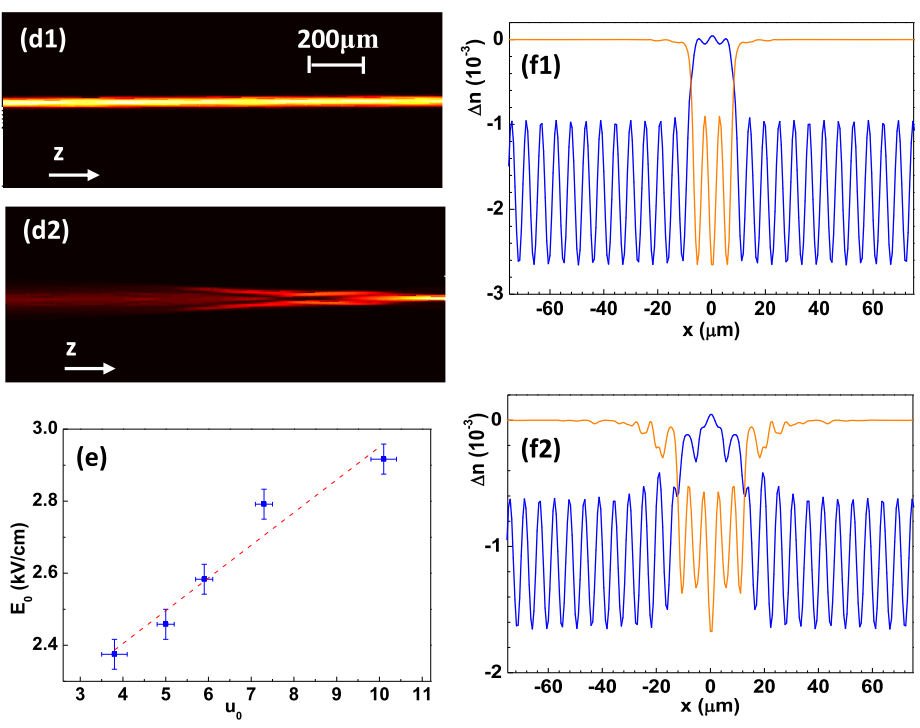

FIG. 3 (color online). Soliton propagation: experimental and numerical results. Observed (a) input, (b) Diffracted, and (c) self-trapped output at applied bias field for (top) 1D beams, with FWHM of $7 \mu \mathrm{m}$ (tight-binding regime), (middle) $22 \mu \mathrm{m}$ (weak-binding regime), and (bottom) 2D beams. In all the cases, continuous localization emerges as confirmed numerically by (d) propagation and (f) associated spatial index of refraction modulation (blue line) compared with just the contribution of the photorefractive response in biased condition (orange line). (e) Experimental relation between normalized intensity and external field for 1D solitons with linear fit (dashed line). 
amplitude and of the beam width. The first statement is verified spanning the experimental soliton parameters $\left(u_{0}, E_{0}\right)$ [Fig. 3(e)] and changing the operational temperature; in particular, we note that even when the lattice amplitude is larger than the standard photorefractive term, the continuous picture remains unchanged. In fact, although we are not able to investigate this regime experimentally because, close to $T_{c}$, the external field needed for soliton formation induces the ferroelectric phase transition, numerical simulations consistently indicate the formation of continuous solitons. Independence from the beam waist is demonstrated launching beams whose size covers several grating periods (weak binding). As reported in the middle row of Fig. 3, a FWHM $=22 \mu \mathrm{m}, u_{0}=2.5$ input beam weakly diffracts when unbiased; it self-focuses up to $8 \mu \mathrm{m}$ in the nonlinear case. Even in this case, the output lacks marked discrete features, as numerically verified. We also demonstrate that what has been achieved occurs in the same spatial lattice geometry for twodimensional solitons; a 2D continuous soliton, $8 \mu \mathrm{m}$ sized, is shown in Fig. 3(c3) at $u_{0}=8.5$. Figure 3(e) reports the relation between normalized intensity and external field for observed 1D solitons; the linear behavior is coherent with the general relationship in centrosymmetric media, even though the slope observed is considerably reduced, this underlining the fundamentally different nature of our present continuous solitons compared to conventional screening solitons [22].

This picture is expected to change if the electro-optical lattice can be decoupled from the photorefractive nonlinear response. Since these two responses act on different time scales, being the electro-optic modulation instantaneous with respect to the photorefractive one, the grating can be decoupled dynamically. This decoupling appears in the first stages of propagation into the lattice nonlinearity and leads to a discrete pattern, as discussed and reported in Fig. 2(d). However, this also happens for transient states after the soliton formation, when the bias field is removed. To further characterize the underlying nonlinear response, we carried out a zero-field readout, as reported in Fig. 4. As the bias field is switched off after the steady state is reached, previously guiding features become antiguiding and vice versa [22]. The effect evolves in time as the now diffracting beam washes out the original soliton-supporting spacecharge distribution. So, in our case, removing the bias field causes the lattice to drop to zero everywhere except for in the region in proximity to the original soliton, where the space-charge field remains unshielded. The beam experiences the local nonlinear lattice and discrete features are now observable. We show the phenomena in Fig. 4 for the dynamics subsequent to the soliton propagation in Fig. 3(a). In the first stages [Figs. 4(a)-4(b)], a discrete delocalized pattern takes place with some "waveguides" more excited than others; numerical analysis at $t=0$, according to the model described in [22], confirms this

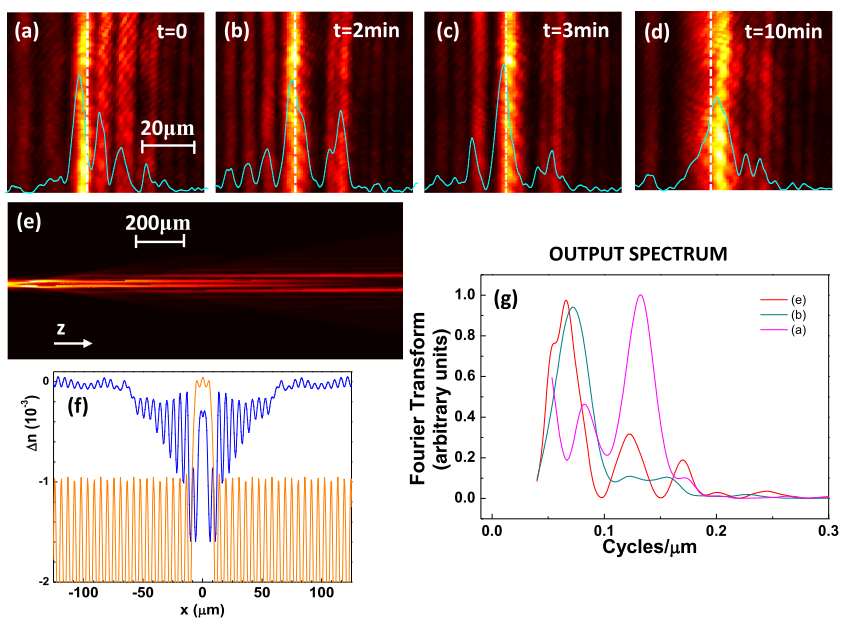

FIG. 4 (color online). Zero-field discrete dynamics after the soliton formation. (a)-(d) Measured time evolution: (a)-(b) discrete delocalized pattern, (c) localization with discrete features and (d) relaxation to the equilibrium. (e)-(f) Numerical results at $t=0$ : (e) discrete propagation and (f) nonlinearity supporting the periodically modulated output light distribution compared with the previous soliton nonlinearity (orange line). (g) Comparison of the spectral properties of (a) (magenta line) and (b) (cyan line) with those of the numerical output in (e) (red line).

behavior, and the resulting beam evolution is reported in Fig. 4(e) with the underlying nonlinearity in Fig. 4(f). The quantitative agreement between experimental and numerical results is made explicit comparing the spectra [Fig. 4(g)]; the excited modes coincide, and the dynamics that cause the spectrum to progressively shed off its peaks, show a consistent transfer of spectral content to the lower frequency component. The characteristic frequencies are identified as the first harmonic of the lattice $K$ and the spatial scale introduced by the beam waist [24]. After this phase, the beam begins to modify the local index pattern and its propagation and interesting transient states can emerge; in Fig. 4(c), we show a transient discrete localization occurring three minutes after the bias field was removed. The nonstationary dynamics cause the localized pattern to spread, ultimately reaching its final equilibrium state, where normal diffraction is almost restored [Fig. 4(d)].

In conclusion, we have demonstrated continuous on-axis soliton propagation in a lattice nonlinearity. The nonlinear photonic lattice is characterized by the electro-optic coupling with the soliton supporting nonlinearity and varies depending on the beam features. These results point out how the periodic properties of a medium can be made to not emerge in the propagating waveform if they are filtered out by a strong interplay between the nonlinear waves and the nonlinear lattice. Discrete effects are shown to appear when the grating is partially decoupled from the photorefractive response, as in the first stages of the biased propagation or in the zero-field dynamics. We also expect this kind of lattice nonlinearity to support interesting soliton interaction 
phenomena [25,26]: specifically, numerical studies (not reported here) indicate that a bound state of $\pi$ out of phase continuous solitons in adjacent lattice sites is possible. In other words, the lattice nonlinearity, as a new periodic optical medium proposed and studied here, opens up interesting perspectives for exploring the physical correlation between nonlinear waves and nonlinear lattices.

Funding from Grant No. PRIN 2012BFNWZ2 and the Sapienza 2014 Awards Project are acknowledged.

*davide.pierangeli@roma1.infn.it

[1] C. Denz, S. Flach, and Y.S. Kivshar, Nonlinearities in periodic structures and metamaterials, Springer Ser. Opt. Sci. 150, 3 (2010).

[2] H. S. Eisenberg, Y. Silberberg, R. Morandotti, and J. S. Aitchison, Diffraction Management, Phys. Rev. Lett. 85, 1863 (2000).

[3] J. Fleischer, G. Bartal, O. Cohen, T. Schwartz, O. Manela, B. Freedman, M. Segev, and N. Efremidis, Spatial photonics in nonlinear waveguide arrays, Opt. Express 13, 1780 (2005).

[4] Y. V. Kartashov, B. A. Malomed, and L. Torner, Solitons in nonlinear lattices, Rev. Mod. Phys., 83, 247 (2011).

[5] H. S. Eisenberg, Y. Silberberg, R. Morandotti, A. R. Boyd, and J.S. Aitchison, Discrete Spatial Optical Solitons in Waveguide Arrays, Phys. Rev. Lett. 81, 3383 (1998).

[6] J. W. Fleischer, T. Carmon, M. Segev, N. K. Efremidis, and D. N. Christodoulides, Observation of Discrete Solitons in Optically Induced Real Time Waveguide Arrays, Phys. Rev. Lett. 90, 023902 (2003).

[7] N. K. Efremidis, S. Sears, D. N. Christodoulides, J. W. Fleischer, and M. Segev, Discrete solitons in photorefractive optically induced photonic lattices, Phys. Rev. E 66, 046602 (2002).

[8] D. Mandelik, H. S. Eisenberg, Y. Silberberg, R. Morandotti, and J.S. Aitchison, Band-Gap Structure of Waveguide Arrays and Excitation of Floquet-Bloch Solitons, Phys. Rev. Lett. 90, 053902 (2003).

[9] D. Mandelik, R. Morandotti, J.S. Aitchison, and Y. Silberberg, Gap Solitons in Waveguide Arrays, Phys. Rev. Lett. 92, 093904 (2004).

[10] C. Lou, X. Wang, J. Xu, Z. Chen, and J. Yang, Nonlinear Spectrum Reshaping and Gap-Soliton-Train Trapping in Optically Induced Photonic Structures, Phys. Rev. Lett. 98, 213903 (2007).

[11] P. Zhang, S. Liu, J. Zhao, C. Lou, J. Xu, and Z. Chen, Optically induced transition between discrete and gap solitons in a nonconventionally biased photorefractive crystal, Opt. Lett. 33, 878 (2008).
[12] Y. Kominis, Bright, dark, antidark, and kink solitons in media with periodically alternating sign of nonlinearity, Phys. Rev. A 87, 063849 (2013).

[13] Z. H. Musslimani, K. G. Makris, R. El-Ganainy, and D. N. Christodoulides, Optical Solitons in PT Periodic Potentials, Phys. Rev. Lett. 100, 030402 (2008).

[14] U. Naether, M. Heinrich, Y. Lahini, S. Nolte, R. A. Vicencio, M. I. Molina, and A. Szameit, Self-trapping threshold in disordered nonlinear photonic lattices, Opt. Lett. 38, 1518 (2013).

[15] A. Ciattoni, C. Rizza, E. DelRe, and E. Palange, Photorefractive solitons embedded in gratings in centrosymmetric crystals, Opt. Lett. 31, 1690 (2006).

[16] D. Pierangeli, J. Parravicini, F. DiMei, G. B. Parravicini, A. J. Agranat, and E. DelRe, Photorefractive light needles in glassy nanodisordered KNTN, Opt. Lett. 39, 1657 (2014).

[17] D. Pierangeli, F. Di Mei, J. Parravicini, G. B. Parravicini, A. J. Agranat, C. Conti, and E. DelRe, Observation of an intrinsic nonlinearity in the electro-optic response of freezing relaxors ferroelectrics, Opt. Mater. Express 4, 1487 (2014).

[18] A. J. Agranat, C. E. M. deOliveira, and G. Orr, Dielectric electrooptic gratings in potassium lithium tantalate niobate, J. Non-Cryst. Solids 353, 4405 (2007).

[19] A. J. Agranat, R. Kaner, G. Perpelitsa, and Y. Garcia, Stable electro-optic striation grating produced by programmed periodic modulation of the growth temperature, Appl. Phys. Lett. 90, 192902 (2007).

[20] L. Wang, H. Tian, X. Meng, H. Chen, Z. Zhou, and Y. Shen, Field-induced enhancement of voltage-controlled diffractive properties in paraelectric iron and manganese co-doped potassium-tantalate-niobate crystal, Appl. Phys. Express 7, 112601 (2014).

[21] T. Imai, S. Toyoda, J. Miyazu, J. Kobayashi, and S. Kojima, Changes in permittivity of KTa1 $-x \mathrm{Nb}_{x} \mathrm{O}_{3}$ crystals induced by electron injection in relation to nonlinear dielectric response, Appl. Phys. Express 7, 071501 (2014).

[22] E. DelRe, B. Crosignani, and P. Di Porto, Photorefractive solitons and their underlying nonlocal physics, Prog. Opt. 53, 153 (2009).

[23] J. Parravicini, D. Pierangeli, F. DiMei, A. J. Agranat, C. Conti, and E. DelRe, Aging solitons in photorefractive dipolar glasses, Opt. Express 21, 30573 (2013).

[24] V. Folli, E. DelRe, and C. Conti, Beam Instabilities in the Scale-Free Regime, Phys. Rev. Lett. 108, 033901 (2012).

[25] G. Fibich, Y. Sivan, and M. I. Weinstein, Bound states of nonlinear Schrödinger equations with a periodic nonlinear microstructure, Physica (Amsterdam) 217D, 31 (2006).

[26] D. Neshev, E. Ostrovskaya, Y. Kivshar, and W. Krolikowski, Spatial solitons in optically induced gratings, Opt. Lett. 28, 710 (2003). 